\title{
Concurrent Cutaneous, Visceral and Ocular Leishmaniasis Caused by Leishmania (Viannia) braziliensis in a Kidney Transplant Patient
}

\author{
Célia MF Gontijo/+ , Raquel S Pacheco*, Fernando Oréfice**, Euler Lasmar***, \\ Eduardo S Silva ${ }^{* * * *}$, Maria Norma Melo $* * * * *$
}

\begin{abstract}
Laboratório de Leishmanioses, Centro de Pesquisas René Rachou-Fiocruz, Av. Augusto de Lima 1715, 30190-002
Belo Horizonte, MG, Brasil *Departamento de Bioquímica e Biologia Molecular, Instituto Oswaldo Cruz-Fiocruz, Rio de Janeiro, RJ, Brasil **Faculdade de Medicina ***Faculdade de Ciências Médicas de Minas Gerais and Hospital Mater Dei, Belo Horizonte, MG, Brasil ****Fundação Educacional de Divinópolis, Universidade do Estado de Minas Gerais, Divinópolis, MG Brasil *****Instituto de Ciências Biológicas, UFMG, Belo Horizonte, MG, Brasil
\end{abstract}

Although cases of leishmaniasis co-infection have been described in acquired immunodeficiency syndrome patients as well as those who have undergone organ transplants, to our knowledge, the present report is the first documented case of simultaneous cutaneous, visceral and ocular leishmaniasis due to Leishmania (Viannia) braziliensis in a transplant patient. The patient had been using immunosuppressive drugs since receiving a transplanted kidney. The first clinical signs of leishmaniasis included fever, thoracic pain, hepatosplenomegaly, leucopenia and anemia. The cutaneous disease was revealed by the presence of amastigotes in the skin biopsy. After three months, the patient presented fever with conjunctive hyperemia, intense ocular pain and low visual acuity. Parasites isolated from iliac crest, aqueous humor and vitreous body were examined using a range of molecular techniques. The same strain of $\mathrm{L}$. (V.) braziliensis was responsible for the different clinical manifestations. The immunosuppressive drugs probably contributed to the dissemination of Leishmania.

Key words: Leishmania braziliensis - cutaneous, visceral and ocular leishmaniasis - kidney transplant

The leishmaniasis are important human diseases in the Americas, producing different clinical manifestations depending on the Leishmania species involved and the host immune response. In the New World, two general forms of the disease can occur: American visceral leishmaniasis (AVL), caused by Leishmania (L.) chagasi and American cutaneous leishmaniasis (ACL), caused by several dermatropic species of Leishmania, of which $L$. (Viannia) braziliensis is the most important. The latter species has the widest geographical distribution, in the New World, and is considered to be responsible for most ACL cases in Brazil. Cases of mucocutaneous leishmaniasis, due to $L$. (V.) braziliensis, have been frequently reported (Marsden 1994).

Leishmaniasis has been identified as an opportunistic infection in patients with alterations in their immune system, especially those with immunodeficiency due to virus infection (Hernández et al. 1995, Ramos et al. 1998) and those who have undergone organ transplants (Borgia et al. 2001). These opportunistic infections, occasionally, produce unusual clinical manifestations. Most published cases are concerned with AVL, in kidney transplant patients, for which identification of the Leishmania species

$\overline{{ }^{+} \text {Corresponding author. Fax }}$ +55-31-3295.3566. E-mail: gontijo@cpqrr.fiocruz.br

Received 28 September 2001

Accepted 5 February 2002 involved had not been performed (Moroni \& Bossi 1995, Berenguer et al. 1998). Reports of cutaneous or mucocutaneous leishmaniasis, in transplant patient, are rare (Golino et al. 1992, Alrajhi et al. 1998).

The cases of ocular leishmaniasis, that have already been reported, are associated with the presence of primary cutaneous leishmaniasis (Roizenblat 1979, Oréfice et al. 1990) or prior cases of AVL (Abdel-Hameed et al. 1991, El Hassan et al. 1998).

To our knowledge, the case described here is the first report of simultaneous cutaneous, visceral and ocular leishmaniasis due to $L$. (V.) braziliensis in a kidney transplant patient.

Case report - A 32-year-old man, from the state of Minas Gerais, Brazil, received a kidney transplant, in 1991, and he has used immunosuppressive drugs (ciclosporine $\mathrm{A}$ at $200 \mathrm{mg} /$ day and prednisone at $5 \mathrm{mg} /$ day) since that time. Since then, the patient has been followed up, in an ambulatory of the Hospital Felicio Rocho, by the transplantation medical care team. In June 1996, the patient showed the first clinical signs of leishmaniasis including fever, pain in the legs and thorax, hepatosplenomegaly, leucopenia and anemia. A series of tests were performed and the possibility of viral infections, such as HIV, CMV and EBV, were put aside. Biopsy of the iliac crest showed amastigote forms indicating that the patient was suffering from AVL. The disease progressed with the appearance of purple pruriginous lesions on the inferior members. A biopsy of the skin with this lesions revealead the presence of Leishmania amastigotes in the tissue. Treatment with Methylglucamine $(850 \mathrm{mg} /$ day $)$ produced an 
improvement in the hematological picture and regression of the hepatosplenomegaly. However, the drug use had to be interrupted after 20 days of treatment because of neurological problems. After three months, the patient presented fever with conjunctive hyperemia, intense ocular pain and low visual acuity. Indirect immunofluorescence titration, for leishmaniasis, was 1:320. Topical application of mydriatic, corticosteroids and acetozolamide $(500 \mathrm{mg} /$ day $)$ associated with amphotericine B lipossomal IV was prescribed. A scan ecography of the right eye (RE) was carried out and revealed diffuse uveitis with edema of the optic disk. Intravitreous treatment with amphotericine $\mathrm{B}(0.010 \mathrm{mg})$ produced a rapid regression of ocular inflammation restoring visual acuity to the RE: HM and LE: 0.6. In February, 1998, the patient returned showing signs of renal failure associated with bilateral endophthamitis. The transplanted kidney was removed and the examination of puncture material from the aqueous humor, vitrous body and bone marrow showed Leishmania parasites. Treatment was initiated with endovenous antimonials, producing regression of ocular inflammation but no improvement in visual acuity. In July, 1998, the patient's condition got worse and treatment with amphotericin B did not provide satisfactory response. The patient, subsequently, experienced heart failure and died.

Characterization of the parasites - Parasites were isolated by puncture of bone marrow, aqueous humor and vitreous body and cultured in NNN/LIT medium. Characterization was carried out using a range of molecular techniques: multi-locus enzyme electrophoresis (MLEE), polymerase chain reaction (PCR), restriction fragment length polymorphisms (RFLP) of kDNA analysis, ramdomly amplified polymorphic DNA (RAPD) and low-stringency single specific primer polymerase chain reaction (LSSPPCR). Isoenzymatic characterization was performed using a system of 13 enzymatic loci (Momen \& Salles 1985). PCR amplification was done using specific primers (B1 5'-GGGGTTGGTGTAATATAG TGG - 3' and B2 5' CTAATTGTGCACG GGGAGG -3') for L. (V.) braziliensis complex (De Bruijn \& Barker 1992). The products were fractionated by electrophoresis in 5\% polyacrylamide gel and silver stained, as previously described (Santos et al. 1993). RFLP analysis was performed according to Pacheco et al. (1986). Maxi and minicircle kDNA fragments were separated in 5-10\% linear polyacrylamide gradient gels after digestion with the restriction enzyme Rsa I. RAPD was performed with four different arbitrary primers: M13 - 40 forward (5' - GTTTTCCCAGTCACGAC - 3'), QG1 (5' - CCATTAGCACCCAAA GCAGACCTCACCC TGTGGAGC-3'), L15996 (5' - CTCCACCATTAGCACC CA AAGC - 3') and another primer of the kit "Ready to Go "Pharmacia (5'-GGTTTC GCTCC-3') following the procedure described by Gomes et al. (1995). LSSP-PCR is performed under low stringency conditions and one specific primer is used in the amplification of the specific fragment. In the present study, both B1 and B2 were used. The reaction was performed in accordance with Pena et al. (1994).

The isolates recovered from the patient, were typed as L. (V.) braziliensis by MLEE (Fig. 1A) and subgenerically classified as Viannia species by specific
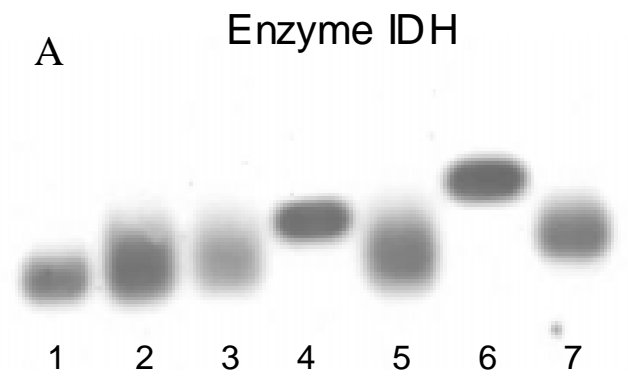

B
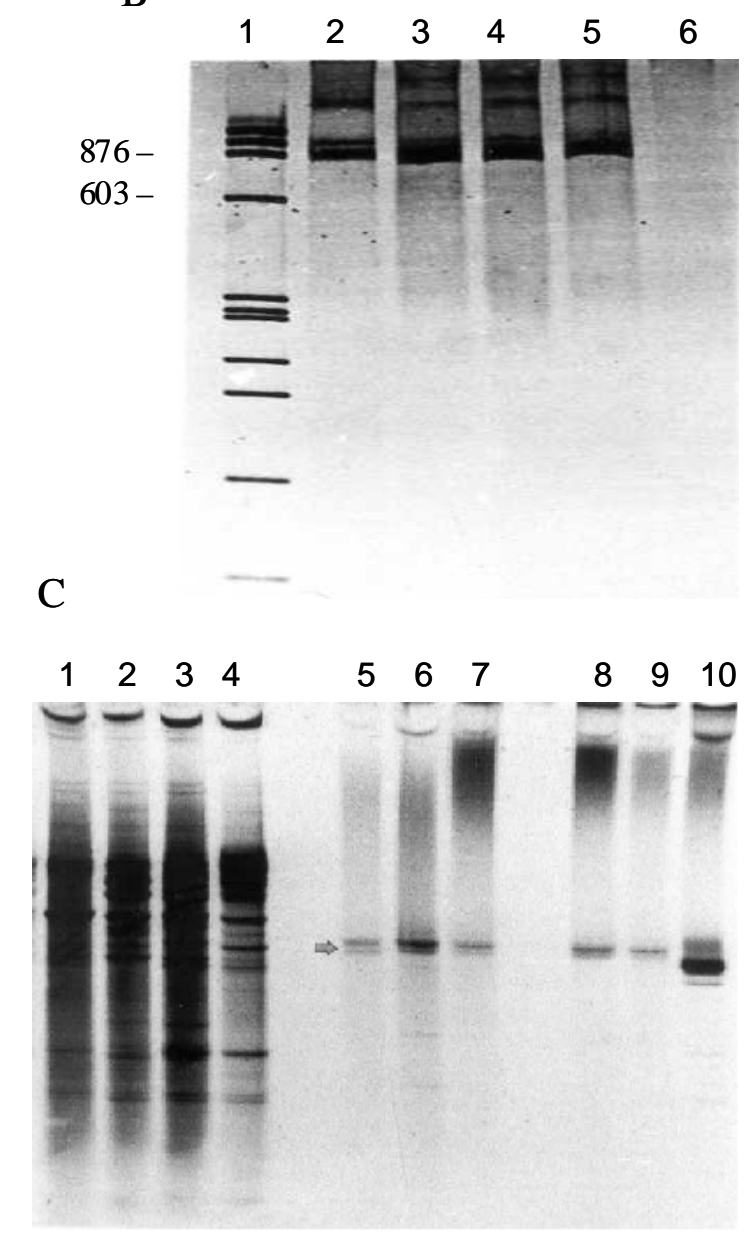

Fig. 1A: electrophoretic profiles of IDH (isocitrate dehydrogenase). Reference strains: lane 1: Leishmania (Viannia) braziliensis; lane 4: $L$. $(L$.$) chagasi; lane 6: L$. $(L$.$) amazonensis and lane 7: L$. (V.) guyanensis; parasites isolated by puncture of vitreous body (lane 2), aqueous humor (lane 3) and bone marrow (lane 5); B: gel electrophoresis of PCR products obtained after amplification using B1 and B2 primers. Lane 1: molecular weight markers $\phi X 174 / \mathrm{Hae}$ III; lane 2: $L$. (V.) braziliensis (reference strain); parasites isolated by puncture of bone marrow (lane 3 ), aqueous humor (lane 4 ) and vitreous body (lane 5); lane 6: $L$. (L.) amazonensis (reference strain); C: kDNA restriction profiles observed after RsaI digestion. Lane 1: $L$. (L.) chagasi (reference strain); lanes 2, 3 and 4: isolates of AVL; parasites isolated by puncture of vitreous body (lane 5), aqueous humor (lane 6) and bone marrow (lane 7); lane 8: isolate of ACL; lane 9: L. (V.) braziliensis (reference strain); lane 10: L. (L.) amazonensis (reference strain) 
PCR (Fig. 1B). No genetic polymorphisms were detected using RAPD, RFLP (Fig. 1C) and LSSP-PCR techniques.

The ability of $L$. (V.) braziliensis to produce a wide spectrum of clinical manifestations could be due to a number of factors, including intrinsic characteristics of the parasite itself and the host immune system.

Experimental infections with different Leishmania species have shown that the number of infected macrophages and amastigotes per macrophage is greater in animals treated with cyclosporin A, which inhibits the production of interleukin IL-1, IL-2 and IFN- $\gamma$ (Olivier et al. 1989). These studies clearly demonstrated that lymphokines or other soluble mediators, produced by T cells, act in controlling infection by Leishmania.

With respect to the human disease, many studies have been performed to further understand the process of resistance and susceptibility to Leishmania infection. The control of leishmaniasis infection, in patients, is due mostly to the cellular immune response, so that reactivation of the disease may occur as a result of immunosuppression (Castés \& Tapia 1998).

Our results revealed that $L$. (V.) braziliensis produced an atypical clinical form of leishmaniasis in the patient, probably due to his immunological state. After organ transplantation he was treated with a combination of cyclosporin A and prednisone, which reduced the production of IL2 and IFN- $\gamma$. This disturbance of cellular immune system decreased his resistance to infections and may have facilitated the spread of Leishmania in others tissues, not normally invaded by this parasite.

The different molecular techniques used here allowed us to confirm that the same strain of parasite was responsible for the different clinical manifestations seen in the patient and demonstrate occurrence of the parasite dissemination. The results reported here reinforce the hypothesis that $L$. (V.) brazilliensis is an opportunistic parasite producing severe manifestations that normally do not occur in immunocompetent individuals. Specific diagnosis of leishmaniasis and characterization of parasites are, therefore, important prerequisites for treatment of immunocompromised patients in Leishmania-endemic areas, due to their increased risk of morbidity and mortality.

\section{REFERENCES}

Abdel-Hameed AA, Hassan MEA, Abdulla KM, el Basha A, Ahmed BO, Mohammedani AA 1991. Two cases of ocular leishmaniasis. Trop Geo Med 43: 91-93.

Alrajhi AA, Saleem M, Ibrahim EA, Gramiccia M 1998. Leishmaniasis of the tongue in a renal transplant recipient. Clin Infect Dis 27: 1332-1333.

Berenguer J, Gómez-Campderá F, Padilla B, Rodriguez-Ferrero M, Anaya F, Moreno S, Valderrabano F 1998. Visceral leishmaniasis (Kala-azar) in transplant recipients. Transplanta- tion 65: 1401-1404.

Borgia F, Vaccaro M, Guarneri F, Manfre C, Cannavo SP, Guarneri C 2001. Mucosal leishmaniasis occuring in arenal transplant recipient. Dermatology 202: 266-267.

Castés M, Tapia FJ 1998. Immunopathology of american tegumentary leishmaniais. Acta Cient Venez 49: 42-56.

De Bruijn MHL, Barker D 1992. Diagnosis of New World leishmaniasis: specific detection of species of the Leishmania braziliensis complex by amplification of kinetoplast DNA. Acta Trop 52: 45-58.

El Hassan AM, Khalil EAG, el Sheikh EA, Zijlstra EE, Osman A, Ibrahim ME 1998. Post kala-azar ocular leishmaniasis. Trans $R$ Soc Trop Med Hyg 92: 177-179.

Golino A, Duncan JM, Zeluff B, De Priest J, McAllister HA, Rodovancevic B, Frazier OH 1992. Leishmaniasis in a heart transplant pacient. J Heart Lung Transplant 11: 820-823.

Gomes RF, Macedo AM, Pena SDJ, Melo MN 1995. Leishmania (Viannia) braziliensis: genetic relationships between strains isolated from different areas of Brazil as revealed by DNA fingerprinting and RAPD. Exp Parasitol 80: 681-687.

Hernández DE, Oliver M, Martínez C, Planas G 1995. Visceral leishmaniasis with cutaneous and rectal dissemination due to Leishmania braziliensis in acquired immunodeficiency syndrome (AIDS). Int J Dermatolol 34: 114-115.

Marsden PD 1994. Mucosal leishmaniasis due to Leishmania (Viannia) braziliensis in Três Braços, Bahia - Brazil. Rev Soc Bras Med Trop 27: 93-101.

Momen H, Salles CA 1985. Enzyme markers for Vibrio cholerae: identification of classical, El Tor and enviromental strains. Trans $R$ Soc Trop Med Hyg 79: 773-776.

Moroni G, Bossi L 1995. Don't forget visceral leishmaniasis in transplant patients. Nephrol Dial Transplant 10: 563-564.

Olivier M, Proulx C, Tanner CE 1989. Importance of lymphokines in the control of multiplication and dispersion of Leishmania donovani within liver macrophages of resistant and susceptible mice. J Parasitol 75: 720-727.

Oréfice F, Pinheiro SRAA, Guedes ACM, Genaro Marra MA, Silveira ILN 1990. Leishmaniose cutânea disseminada iridociclite bilateral não granulomatosa. Isolamento de Leishmania no humor aquoso. Arq Bras Oftal 53: 77-79.

Pacheco RS, Lopes UG, Morel CM, Grimaldi Jr G, Momem H 1986. Schizodeme analysis of Leishmania isolates and comparison with some phenotypic techniques. In JA Rioux, Leishmania: Taxonomy and Phylogeny, IMEEE, Montpellier, p. 57-65.

Pena SDJ, Barreto G, Vago AR, De Marco L, Reinach FC, Dias Neto E, Simpson AJ 1994. Sequence-specific 'gene signatures' can be obtained by PCR with single specific primers at low stringency. Proc Natl Acad Sci 91: 1946-1949.

Ramos A, Portero JL, Gazapo T, Yebra M, Portero F, Martin T 1998. Visceral leishmaniasis in immunocompromised patients. An Med Interna 15: 301-304.

Roizenblat J 1979. Intersticial keratitis caused by American (mucocutaneous) leishmaniasis. Am J Ophthalm 87: 175-179.

Santos FR, Pena SDJ, Epllen JT 1993. Genetic and population study of a Y-linked tetranucleotide repeat DNA polymorphism with a simple non-isotopic technique. Humans $G e$ netics 90: 655-656. 
754 Leishmaniasis in a Kidney Transplant - Célia MF Gontijo et al. 